\title{
Temporal Subtraction of Thorax CR Images
}

\author{
Dirk Loeckx, Frederik Maes ${ }^{\star}$, Dirk Vandermeulen, and Paul Suetens \\ Medical Image Computing (Radiology-ESAT/PSI), Faculties of Medicine and \\ Engineering, University Hospital Gasthuisberg, Herestraat 49, B-3000 Leuven, \\ Belgium. Dirk.Loeckx@uz.kuleuven.ac.be
}

\begin{abstract}
We propose a non-rigid registration algorithm for temporal subtraction of thorax CR-images. The images are deformed using a statistically trained B-spline deformation mesh based on principal component analysis of a training set. Optimization proceeds along the transformation components rather then along the individual spline coefficients, using pattern intensity as the criterion. The algorithm is trained on a set of 30 lung pairs and verified on a set of 46 lung pairs. In $96 \%$ of the cases the achieved registration is subjectively rated to be adequate for clinical use.
\end{abstract}

\section{Introduction}

Radiologists commonly compare a current chest radiograph with a previous one in order to facilitate the detection of new abnormalities. These abnormalities can be caused for instance by pulmonary nodules, interstitial infiltrates, pleural effusions or cardiomegaly. Because of the limited quality of the exposure and the subtlety of the interval changes, abnormalities are often difficult to detect, especially if they overlap with anatomical structures such as ribs, vessels, heart or diaphragm.

Temporal subtraction is a technique in which a previous recording of the same patient is subtracted from the current recording, after proper alignment and warping. With temporal subtraction, the visibility of interval changes increases significantly. In the ideal case, all structures that didn't change over time disappear in the difference image. This includes not only the ribs but also the other structures present in the lungs. Besides, abnormalities unchanged since the previous recording largely disappear, whereas a change in size becomes clearly visible. Thus, temporal subtraction emphasizes the differences between the previous and the current recording, caused by new or changed abnormalities.

The major challenge to create a good subtraction image is a correct registration of both images. The registration has to compensate for a difference in pose, recording setup and inhalation. This registration is complicated because the $2 \mathrm{D}$ warp of the X-ray images has to compensate for the $3 \mathrm{D}$ transformation of the thorax and lungs. Since the 2D X-rays are projections of the real-world

\footnotetext{
* Frederik Maes is Postdoctoral Fellow of the Fund for Scientific Research - Flanders (FWO-Vlaanderen, Belgium).
} 
$3 \mathrm{D}$ structure there is no single solution that maps all corresponding structures on each other.

Within this article, we propose a new warping scheme. The goal of the warp is to reduce misalignment artifacts in the difference image of the X-ray images. To minimize those artifacts, a non-rigid registration is performed with pattern intensity [1] as image-based similarity measure. We assume that only a small part of the image will have changed fundamentally and thus that the abnormalities have only a minor influence on the criterion.

The possible causes for the transformation mentioned above constrain the registration space. Thus, the warps between different image pairs are strongly correlated. To incorporate this knowledge in the registration algorithm, the search space is limited to the most common modes of transformation as obtained from a statistical analysis of a training set of registered image pairs.

Registration quality is judged by a human observer, rating the presence of residual rib-related artifacts in the subtraction images.

\section{Methods}

The registration and warping of one radiograph to another necessitates three important parts: a way to model the transformation, a registration criterion to judge the goodness of fit and an optimization strategy.

\subsection{Spline Deformation Model}

Because of their limited support, scalability and multiresolution properties, a 2D tensor product B-spline was chosen to model the transformation [2]. Our experiments with manual registration of temporal pairs of chest radiographs indicate that a $2 \mathrm{D}$ tensor product B-spline of the third order with $6 \times 6$ knots ( 2 knots of multiplicity 3 in each dimension) and thus $3 \times 3$ control points allows sufficient degrees of freedom to achieve an adequate registration of the ribs. The spline control point configuration is illustrated in Fig. 1. As different control points for the horizontal and vertical transformation field are needed, 18 parameters are used.

To further reduce the search space and improve the specifications of the optimization a statistical analysis, principal component analysis (PCA), is performed on a manually registered training set 3 . This way we can reduce the number of degrees of freedom by only retaining the most significant modes of variation as observed in the training set. Affine translation and scale modes are merged with the PCA modes into a unified approach. The mean and standard deviation of all modes of variation are also obtained.

\subsection{Registration Criterion}

Some anatomical features outside the lung fields, such as the clavicle, mediastinum or parts of the limbs, transform independently of the lung field, and 
their presence in the image may interfere with finding a proper registration for the lung field itself. Hence, the lung field is segmented prior to registration and non-lung regions are excluded when computing the registration criterion. This improves registration robustness and at the same time increases the calculation speed. Lung field segmentation is achieved using a variation of the Active Shape Model segmentation algorithm with optimal image features [4. This results in a region of interest (ROI) containing both lung fields, including the lateral lung boundaries and excluding the mediastinum. Two example images with overlaid ROI's can be seen in Fig. 1] The ROI of the first image, which is warped towards the second image, undergoes the same transformation as the first image and is overlaid on the ROI of the second image. The registration criterion is computed over all pixels in the union of both ROI's.

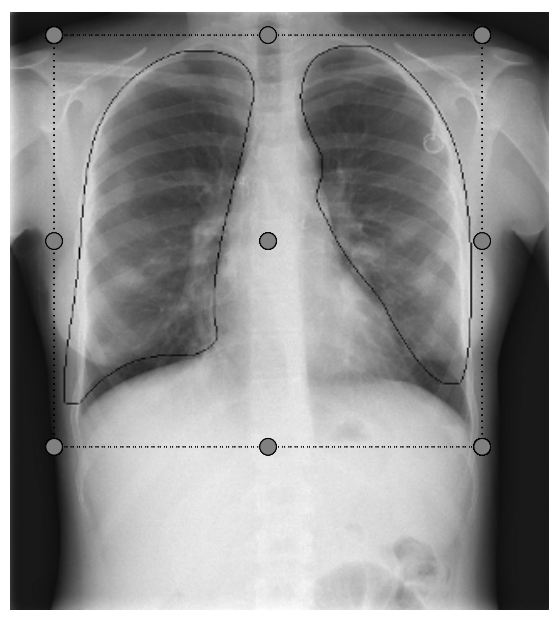

(a)

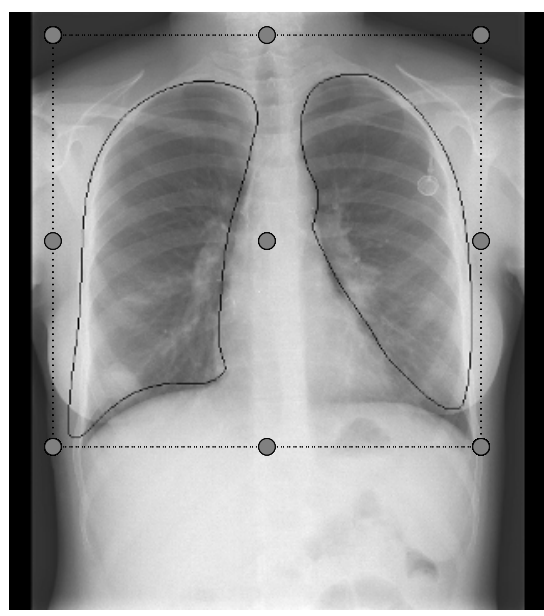

(b)

Fig. 1. Pair of digital thorax radiographs to be registered, with $3 \times 3$ spline control point mesh overlaid and automatically segmented lung field ROI's.

Different registration criteria were tested, but pattern intensity [1]

$$
P_{r, \sigma}\left(I_{\text {diff }}\right)=\sum_{x, y} \sum_{r \leq r_{\max }} \frac{\left(I_{\text {diff }}(x, y)-I_{\text {diff }}(v, w)\right)^{2}}{\sigma^{2}+\left(I_{\text {diff }}(x, y)-I_{\text {diff }}(v, w)\right)^{2}}
$$

with $I_{\text {diff }}$ the difference image, radius $r=\sqrt{(x-u)^{2}+(y-v)^{2}}$ and threshold $\sigma$, was found to be best behaved around the registration solution. PI measures the remaining artifacts in the difference image. The radius $r_{\max }$ selects the scale of the artifacts, while the threshold $\sigma$ serves to equalize their contribution. Roughly 
spoken, artifacts smaller than $r$ are considered as noise, while artifacts with an intensity difference larger than $\sigma$ will have the same contribution to the penalty. We have used $r_{\max }=3.5$ pixels and $\sigma=128$, but these values were not critical.

\subsection{Optimization}

Because of the nature of the lung images, the cost function is not smooth and has multiple optima. As standard optimization algorithms are found unreliable for our problem, a multi resolution fast simulated annealing [5] optimization strategy is chosen. The transformation is parameterized by the modes of variation as obtained from the principal component analysis rather then over the spline control points directly. Also the boundaries of the optimization parameters are expressed in units of standard deviation. An example of the course of the cost function around the optimum can be seen in Fig. 2 .
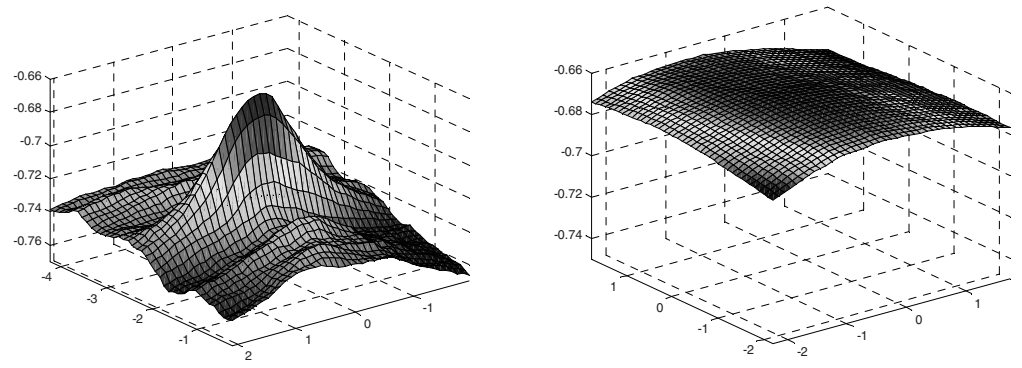

Fig. 2. Dependence of cost-function around the optimum on (left) translations (XY) and (right) the first two PCA modes.

Initially, the centers of mass of both ROI's are horizontally aligned and the lung tops vertically. Registration then occurs in two steps. In the first step, we perform an affine registration using only the translation and scale modes. In the second step, we allow $t$ degrees of freedom, where $t$ can be any number between 3 to 18 , i.e. $t$ varies from affine to full spline registration. For the first step the original images are resized to $64 \times 64$ pixels, the second step is performed on images of $128 \times 128$ pixels.

The transformation is passed on from one resolution to another by scaling the transformation mesh rather than the transformed image. Images are resampled and morphed using B-spline interpolation [6,7,8, B-spline interpolation yields a very good sub-pixel precision, allowing for the small image processing sizes.

\subsection{Implementation}

Development and implementation of the algorithm was performed in Matlab, focusing rather on the quality of registration than on the performance. The 
registration software is implemented and ran on a Dell Precision 530 workstation with dual Xeon 2.2 Ghz/512k processors in Matlab 6.5 release 13 for Linux 9], while the most time-critical procedures are coded in $\mathrm{C}++$.

\section{$2.5 \quad$ Validation}

As is often the case with real data sets, a gold standard is not available for the validation of the registration. Most frequently, validation is still performed by a human observer valuing each registration. Therefore, the registered image pairs were evaluated using a five-point rating scale. This scale is identical to the scale used by Ishida et al [10, as shown in table 1.

Table 1. Five-point rating scale [10].

\begin{tabular}{ll}
\hline 1. Very Poor & $\begin{array}{l}\text { Most ribs are not registered and appear in } \\
\text { the entire intercostal space; }\end{array}$ \\
2. Poor & $\begin{array}{l}\text { Most ribs are not well registered and appear } \\
\text { in half of the intercostal space }\end{array}$ \\
3. Adequate & $\begin{array}{l}\text { Most ribs are well registered, with some mi- } \\
\text { nor misregistration error }\end{array}$ \\
4. Good & $\begin{array}{l}\text { Most ribs are almost completely registered } \\
\text { with some very minor misregistrations }\end{array}$ \\
5. Excellent & All ribs are perfectly registered
\end{tabular}

The subtraction images scored as 1 or 2 need further improvement before they can be used in clinical practice. The images scored as 3 or better are good subtraction images and adequate for clinical use. An example of each set can be seen in Fig. 3.

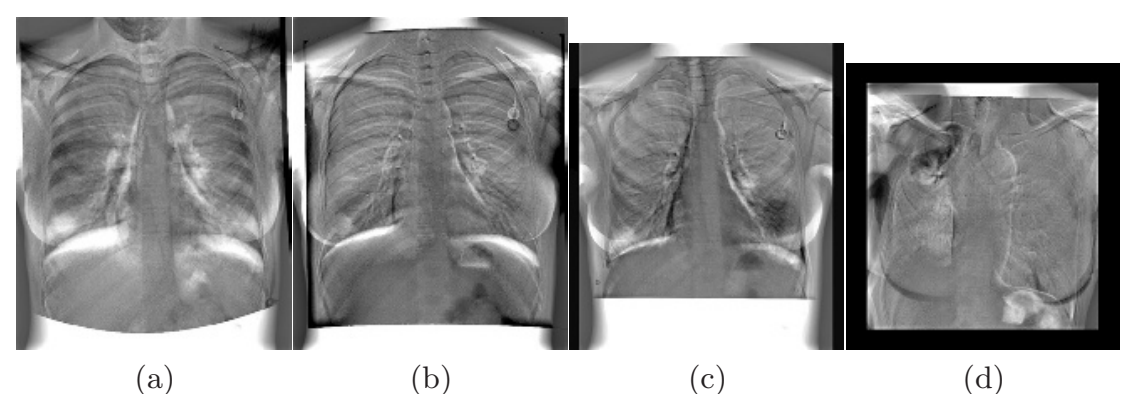

Fig. 3. Typical examples of (a) very poor, (b) adequate, (c) good and (d) excellent registrations. See Table 1 for a description of the rating scale. 


\section{Results}

The algorithm described above was applied to the temporal registration of thorax CR (computed radiography) images. The images are extracted from the PACS (Picture Archiving and Communication System) of the University Hospital Gasthuisberg of the Katholieke Universiteit Leuven in Leuven, Belgium. They were recorded for different clinical studies on different X-ray imaging systems using normal recording settings. The resolution and gray level range of the $\mathrm{CR}$ images are about $2500 \times 2500$ pixels and 12 bits respectively. No special precautions were taken to ensure the pictures were recorded under the same recording settings. This corresponds to the clinical practice where radiologists compare a current picture with any similar recording of the same patient without constraints on its origin.

The algorithm is developed and trained on a set of 30 image pairs, and validated on a set of 46 other image pairs. Not all image pairs are disjoint. In the training set, if more than two radiographs of the same person are available, all possible pairs are used (i.e. three radiographs lead to three pairs, four radiographs lead to six pairs). In the validation set, if more than two radiographs of the same person are available, one image is randomly chosen and paired with every other image of this person. The training set is used to train the active shape model segmentation with optimal features and to train the statistical spline deformation models. During the registration, $t=12$ degrees of freedom were used.

Registration times are relatively high with an average duration of about 150 seconds per pair. The segmentation is performed separately and takes about 90 seconds per image.

The obtained subtraction images were rated by the author on a normal computer screen. In order to use as much of the available range of the display system as possible, the histogram of the difference image in the ROI is extended over the available gray value scale. Points outside the ROI are mapped in the same way, values outside the ROI interval become white or black. Also, although it is only optimized over the rectangle defined by the spline knots, the transformation is expanded to the edges of the image. As this is an extrapolation it is only a rough estimation, but, to our opinion, it's favorable over an abrupt change in the image at the edge of the spline area.

The number of cases in each rating category is shown in table 2 Less then $5 \%$ of the registered pairs is inadequate for clinical use. Most of the registrations are rated 'good'

Table 2. Number of cases in each subjective rating category.

\begin{tabular}{|c|c|c|c|c|c|}
\hline & $\begin{array}{c}1 \\
\text { Very Poor }\end{array}$ & $\begin{array}{c}2 \\
\text { Poor }\end{array}$ & $\begin{array}{c}3 \\
\text { Adequate }\end{array}$ & $\begin{array}{c}4 \\
\text { Good }\end{array}$ & $\begin{array}{c}5 \\
\text { Excellent }\end{array}$ \\
\hline \# of images & 1 & 1 & 12 & 30 & 2 \\
\hline$\%$ of total & $2 \%$ & $2 \%$ & $26 \%$ & $65 \%$ & $5 \%$ \\
\hline
\end{tabular}




\section{Discussion}

For the lung segmentation, the use of active shape models with optimal features is favorable over standard active shape models because not all landmarks are positioned on a clear edge in the image. As we only need a rough indication of the lung field, the precision of the segmentation algorithm is largely sufficient for the current application. Yet in about $10 \%$ of the cases, the lower part of the lung is missed due to breast artifacts. The number of failures can be reduced using a larger training set.

For the registration, the number of misregistration errors occurring is dependent on the amount of time spent in the simulated annealing optimization step. Simulated annealing is, due to its random approach, not a very efficient optimization method, but given sufficient time, it will converge to the global optimum. A final optimization step consisting of a downhill simplex algorithm over the spline coefficients was also tested, but the added precision it yields is negligible.

Previous work on temporal subtraction of lung fields has been performed by Kano et al [1]. After initial registration of the images, they calculate regional shift vectors and fit a two-dimensional polynomial through them. This scheme was later improved by Ishida et al [10. Starting from images obtained on a Fuji-CR system, they get a good overall performance, but some misregistration errors still occur. Subtraction is also used in other fields like digital subtraction angiography (DSA), other angiographies, breast cancer detection, multiple sclerosis detection in brain MRI images, etc.

Comparing our results with the results obtained by Ishida et all we notice that the performance of both algorithms is about the same. Because they allow more degrees of freedom, the algorithm of Ishida will be more precise for small details. But this also means that local artifacts that do not move together with the ribs, like the clavicle or the mediastinum, will lead to local misregistrations. Due to the spline model and the learned transformations, our algorithm looks at a more global scale and thus will be less precise for small details but also les prone to local inaccuracies.

The incorporation of statistical information in the transformation reduces the number of modes for the transformation without constraining any physical mode. In our case we were able to reduced the number of modes from 18 to 12 modes, a reduction of $33 \%$. This method can be extended to more localized transformations or to a higher accuracy by increasing the number of knots of the transformation spline, although the number of training images should increase accordingly.

Performing a statistical analysis of a deformation field was previously introduced by Nastar et al [12] and Rueckert et al [13]. The former incorporated the dimensional reduction provided by a principal component analysis (PCA) in the Lagrangian equilibrium equation applied to face matching. The latter worked out the concept of statistical deformation models (SDM) to construct an atlas of the brain, containing information about the average anatomy as well as its variability across a population of subjects. 
New to our approach is the application of PCA-guided registration directly to the spline coefficients and to medical images. Here PCA is not only useful to increase the speed, but also an important surplus to get an appropriate registration. Also new is the unification of the affine and PCA components of the transformation into a single framework. This approach leads to a reduction in the needed number of degrees of freedom 3 .

To improve the robustness of the optimization, two options are open, either enhancing the optimization method or developing a better criterion, i.e. smoother and with less local optima. Our experience show that further reducing the number of degrees of freedom is not a good option. Because of the limited extend of the optimum, the correspondence between the static image and the warped image should be sufficient before the criterion is trapped in it. E.g., if the breathing transformation isn't right, there will be no good optimum for the translation. Further improvement of the accuracy could be achieved by adding a final registration step with a refined B-spline mesh.

\section{References}

1. Weese, J., Buzug, T.M., Lorenz, C., Fassnacht, C.: An approach to 2D/3D registration of a vertebra in 2D X-ray fluoroscopies with 3D CT images. In: Proc. CVRMed/MRCAS. (1997) 119-128

2. de Boor, C.: A Practical Guide to Splines. Springer-Verlag, New York (1978)

3. Loeckx, D., Maes, F., Vandermeulen, D., Suetens, P.: Non-rigid image registration using a statistical spline deformation model. In: Proc. IPMI. (2003) accepted.

4. van Ginneken, B., Frangi, A., Staal, J., ter Haar Romeny, B., Viergever, M.: Automatic detection of abnormalities in chest radiographs using local texture analysis. $\mathrm{PhD}$ thesis, Universiteit Utrecht (2001)

5. Ingber, L.: Very fast simulated annealing. Mathematical and Computer Modelling 12 (1989) 967-973

6. Unser, M., Aldroubi, A., Eden, M.: B-spline signal processing: Part I-theory. IEEE Trans. Signal Processing 41 (1993) 821-832

7. Unser, M., Aldroubi, A., Eden, M.: B-spline signal processing: Part II-efficient design and applications. IEEE Trans. Signal Processing 41 (1993) 834-848

8. Unser, M.: Splines: A perfect fit for signal and image processing. IEEE Signal Processing Mag. 16 (1999) 22-38

9. MATLAB. Version 6.5.0.180913a (R13) (2002)

10. Ishida, T., Katsuragawa, S., Nakamura, K., MacMahon, H., Doi, K.: Iterative image warping technique for temporal subtraction of sequential chest radiographs to detect interval change. Medical Physics 26 (1999) 1320-1329

11. Kano, A., Doi, K., MacMahon, H., Hassell, D.D., Giger, M.: Digital image subtraction of temporally sequential chest images for detection of interval change. Medical Physics 21 (1994) 453-461

12. Nastar, C., Moghaddam, B., Pentland, A.: Generalized image matching: Statistical learning of physically-based deformations. In: Proc. ECCV. (1996)

13. Rueckert, D., Frangi, A., Schnabel, J.: Automatic construction of 3D statistical deformation models using non-rigid registration. In: Proc. MICCAI. (2001) 77-84 